\title{
CUBIERTA NIVAL, PERMAFROST Y FORMACIÓN DE FLUJOS SUPERFICIALES EN UN TALUD DETRÍTICO DE ALTA MONTAÑA (CORRAL DE VELETA, SIERRA NEVADA, ESPAÑA)
}

\section{M. TANARRO GARCÍA ${ }^{(1)}$, D. PALACIOS ESTREMERA ${ }^{(1)}$ J. J. ZAMORANO OROZCO( ${ }^{(2)}$, A. GÓMEZ ORTIZ ${ }^{(3)}$}

(1) Departamento de Análisis Geográfico Regional y Geografía Física, Universidad Complutense de Madrid

(2) Instituto de Geografía. Universidad Nacional Autónoma de México (UNAM)

(3) Departamento de Geografía Física y Análisis Geográfico Regional, Universidad de Barcelona

Correo electrónico de contacto: pace@ghis.ucm.es

RESUMEN. En este trabajo se estudia el origen de una serie de movimientos en masa, del tipo flujo superficial, ocurridos en la última década en el talud detrítico de la base de la pared septentrional del Pico del Veleta en Sierra

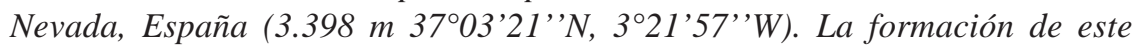
tipo de coladas se relaciona con la acción geomorfológica de la nieve y con la degradación de hielo en el subsuelo o permafrost. Con este objetivo, se ha realizado un control anual (periodo 1998-2008) de la permanencia y extensión de la cubierta de nieve mediante la toma de fotografías oblicuas a finales del verano y se han observado y cartografiado las variaciones morfológicas del talud en esos años. Los resultados indican que la tendencia reciente de la acumulación y permanencia de la nieve a finales del verano se ha reducido de manera global. Por este motivo, en los últimos años durante los meses estivales el talud permanece libre de la cubierta de nieve, cuya consecuencia más inmediata está siendo la degradación del permafrost existente. La comparación entre el comportamiento de la nieve y los años de formación de flujos superficiales en el talud ha permitido detectar el momento y lugar de la aparición de estos procesos. Los flujos superficiales se forman especialmente en años de un rápido y temprano deshielo de la cubierta nival y lo hacen en los frentes de los neveros más persistentes. Las características sedimentológicas de la masa de derrubios, que contiene abundante material fino, permite en esos momentos la desestabilización de la parte superficial del talud y el despegue y desplazamiento de una delgada capa de material, que tan solo arrastra partículas finas, cantos y pequeños bloques. 


\begin{abstract}
This study examines the origin of a series of mass movements called skin flows occurring on a scree slope located at the foot on the north wall of Pico del Veleta in Sierra Nevada, Spain (3398 m, 37 03'21' 'N, 3²1' 57' 'W). The genesis of these flows is contrasted to the geomorphologic action of snow and the degradation of buried ice masses or permafrost. Oblique photography was used during late summer, yearly monitoring of snow cover duration and surface area from 1998-2008 and the variations of talus morphology. The results show a recent trend in reduced snow cover duration in late summer. The talus is free of snow cover during this season and permafrost degradation in some sections of the talus has accelerated. The contrast between snow cover evolution and skin flows formation indicates when and where the processes occurred. Skin flows are most apt to form on the downslope side of long-lasted snow patches when snow melts rapidly. The sedimentologic characteristics of the detritus material containing large amounts of fine sediment is often responsible for triggering superficial talus destabilization and subsequent detachment and displacement of a thin layer of materials that drags fine sediment, stones and small boulders with it.
\end{abstract}

Palabras clave: degradación del permafrost, talud de derrubios, flujos superficiales, permanencia de la cubierta de nieve, Pico de Veleta (Sierra Nevada).

Key words: permafrost degradation, detritic talus, skinflows, snow cover duration, Veleta Peak (Sierra Nevada).

Enviado el 6 de octubre de 2009 Aceptado el 17 de diciembre de 2009

\title{
1. Introducción
}

La cubierta de nieve puede desempeñar un doble papel geomorfológico en relación, por un lado, con su espesor y duración y, por otro, con los fenómenos erosivo-sedimentarios asociados a su deshielo y/o fusión. En el primer caso, la presencia de una cobertera nival de cierto espesor, al menos de 0,8-1 m, actúa como un eficaz aislante térmico entre la interfaz suelo-atmósfera atenuando drásticamente el balance de energía en la superficie del suelo (Haeberli, 1973). La nieve, debido a su alto albedo, refleja la mayor parte de la radiación solar incidente. Por otro lado, por su naturaleza porosa, es un eficiente aislante térmico. De este modo una cubierta nival que se prolongue sobre el suelo a lo largo de los años, lo aísla de las variaciones térmicas la atmósfera y evita, en caso de que exista, la degradación del permafrost (Goodrich, 1982; Hall, 1985; Funk y Hoelzle; 1992; Hoelzle, 1996; Heggem et al., 2001; Chueca y Julián, 2004). Por el contrario, la acción geomorfológica más importante relacionada con la nieve se produce a raíz de su deshielo, lo que da lugar a movimientos en masa de distinta tipología (Rapp, 1960; Jahn, 1967; Benedict, 1970; Caine, 1976; Soderman, 1980; White, 1981; Akerman, 1984; Strömquist, 1985; Harris, 1987; Rapp y Nyberg, 1988; Nyberg, 1991), que son 
especialmente importantes cuando la fusión es rápida y afecta a materiales sin consolidar (Christiansen, 1998; Palacios et al., 2003; Andrés y Palacios, 2004).

En diversas altas montañas europeas se está detectando la degradación del permafrost ante el calentamiento global, lo que puede dar lugar al aumento de los procesos naturales de tipo catastrófico (Harris et al., 2003), como sucede con la desestabilización de taludes detríticos de fuerte pendiente (Andersland y Ladanyi, 2004). Se han señalado algunos tipos de movimientos en masa sobre taludes asociados a la fusión de permafrost: flujos, como solifluxión, deslizamientos superficiales (skinflows), flujos bimodales (bimodal flows) flujos retrogresivos múltiples (multiple retrogressive flows) y flujos de derrubios (debrisflows); deslizamientos como los retrogresivos múltiples (multiple retrogressive slides), los de bloques (block slides) y los rotacionales (rotational slides); y, finalmente, desprendimientos (McRoberts y Morgenstern, 1974; Lewkowicz, 1988). En concreto, los flujos superficiales se han asociado indistintamente con la fusión rápida de la nieve y con la degradación de niveles de permafrost (Jahn, 1967; Nyberg, 1991; Rapp y Nyberg, 1988; Strömquist, 1985; McRoberts y Morgenstern, 1974, Caine, 1976).

El Pico del Veleta (3.398 m, 3703'21'’N, 321'57' 'W) en su vertiente septentrional, donde se localiza el circo denominado Corral del Veleta (Fig.1), retiene uno de los últimos reductos de permafrost del sur de Europa (Gómez et al., 1999; 2001). En este circo, la permanencia de la nieve a lo largo de la última década ha sido cada vez más

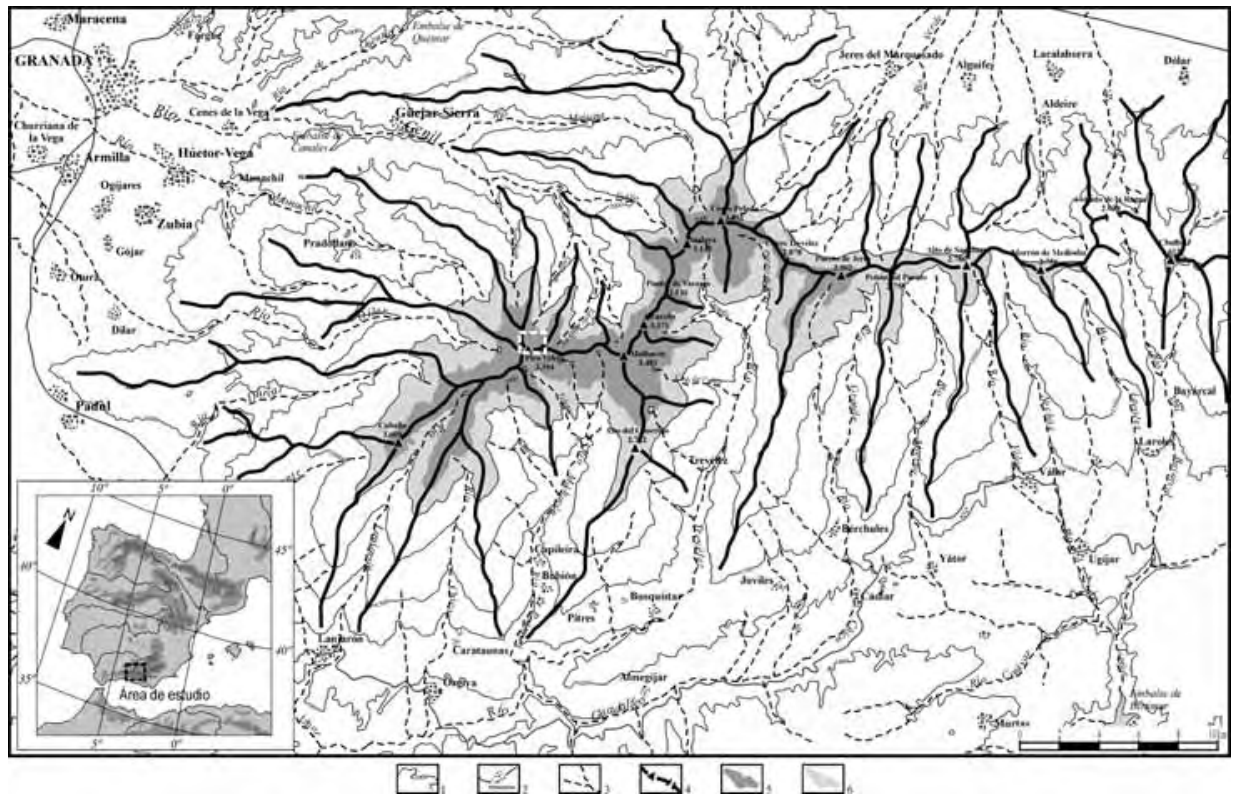

Figura 1. Localización del área de estudio. 1. Curvas de nivel; 2. Núcleos de población; 3. Red hidrográfica; 4. Divisorias; 5. Áreas por encima de los $2.500 \mathrm{~m}$ de altitud; 6. áreas por encima de los $2.800 \mathrm{~m}$ de altitud. 


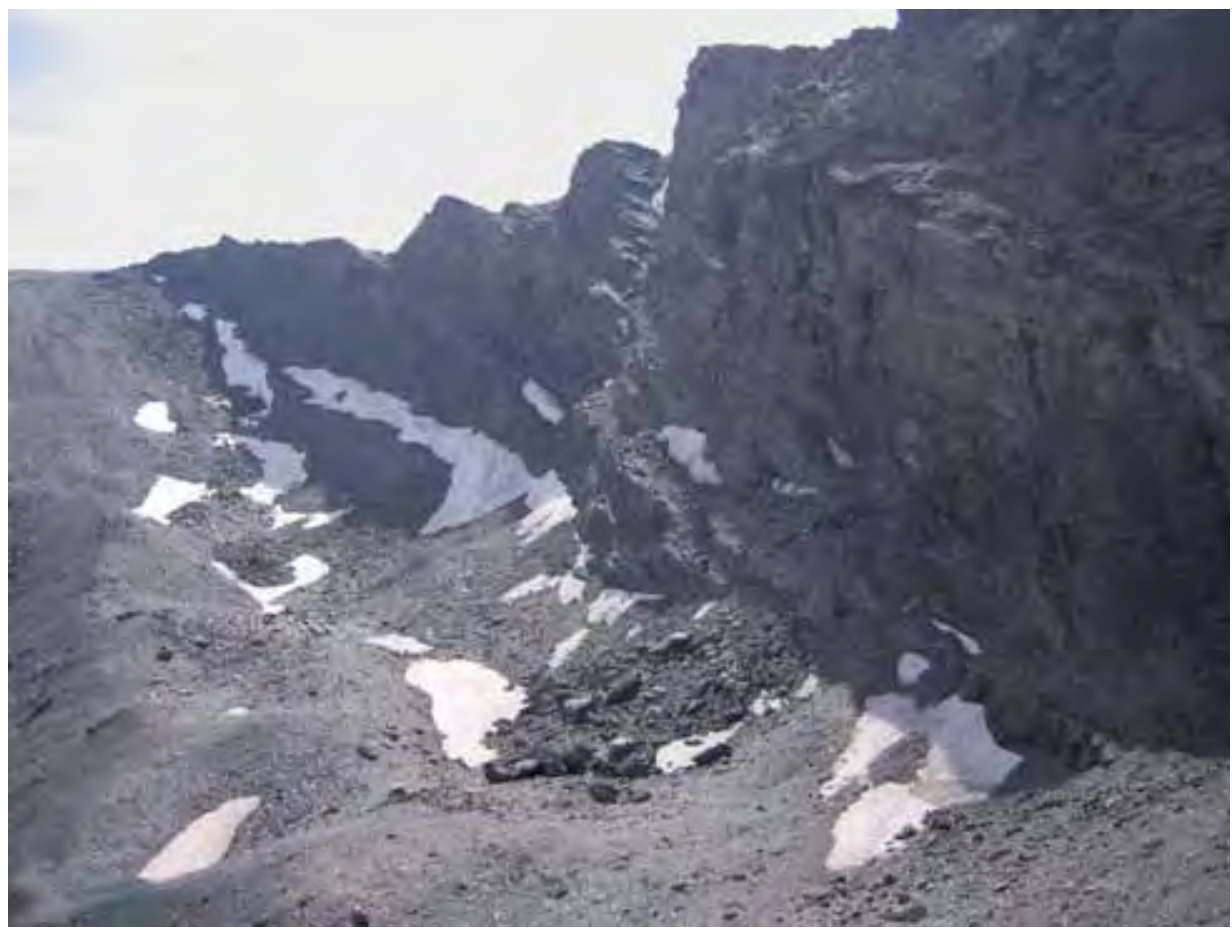

Figura 2. Fotografía que muestra los elementos morfológicos característicos del conjunto del Corral del Veleta: la pared, el fondo del circo, el cordón morrénico externo y el talud detrítico.

limitada (Gómez et al. 2008). El talud, situado al pie de la pared norte que delimita el Pico del Veleta (Fig. 2), muestra una activa dinámica geomorfológica, según se ha detectado a lo largo de trabajos de campo realizados en los últimos diez años (1998-2008), en los que se ha podido observar el desarrollo de frecuentes movimientos en masa, entre los cuales destaca la formación de flujos rápidos pero muy superficiales, junto con deslizamientos rotacionales (Gómez et al., 2003). Esta creciente actividad geomorfológica del talud podría estar relacionada con la desprotección de la nieve durante el período estival en estos últimos años y la consecuente degradación del permafrost subyacente.

En este contexto, el objetivo de este trabajo es analizar la importancia de la acción geomorfológica de la nieve y de la degradación del permafrost como factores desencadenantes de una serie de flujos superficiales ocurridos en el talud de la pared norte del Pico del Veleta, caracterizados por el despegue y posterior movimiento de una delgada capa de derrubios, hasta formar una lengua de decenas de metros de longitud sobre el plano inclinado de la superficie topográfica del talud (McRoberts y Morgenstern, 1974; Lewkowicz, 1988). Con este objetivo, y durante el período 1998-2008, se ha evaluado la permanencia de la nieve sobre el talud, se han cartografiado sus variaciones morfológicas, en particular los nuevos flujos formados y se han determinado las relaciones existentes entre nieve y desplazamientos. También, se ha prestado especial atención a delimitar la localización de 
estos flujos y su posible relación con la existencia de permafrost, así como su perviviencia o desaparición en años posteriores a su formación.

\section{El área de estudio}

La pared norte del Pico del Veleta desciende unos $300 \mathrm{~m}$ verticalmente hasta la base del Corral del Veleta, situada aproximadamente a 3.060 m (Fig. 2). La pared está constituida por rocas metamórficas, con predominio de micaesquistos feldespáticos de estructura laminada del manto del Veleta (Puga et al., 1974), afectados por profundas fracturas recientes, atribuidas a fenómenos neotectónicos (Sanz de Galdeano y Lopéz, 1999) y a la descompresión del hielo glaciar que colmó buena parte de este circo durante el Pleistoceno (Gómez et al., 1996). La pared está escalonada por la presencia de una serie de estrechas repisas estructurales inclinadas hacia el NNW, en las que se acumulan importantes cantidades de clastos de gravedad. Un talud detrítico dispuesto de oeste a este enlaza esta pared con el último escalón estructural que da lugar al fondo del Corral, el cual está delimitado en su borde externo por cordones morrénicos finipleistocenos y holocenos (Fig. 2). El talud está constituido por abundantes derrubios que proceden de la destrucción de la pared (meteorización intensa por gelifracción, caída libre y ocasionales desprendimientos de gran entidad) (Gómez et al., 2003). La forma del talud es escalonada pues los derrubios se acumulan cubriendo repisas estructurales. También el talud está excavado por pequeños nichos nivales y por las cabeceras de cicatrices de despegue de deslizamientos rotacionales y de lenguas de reptación.

Desde un punto de vista sedimentológico el talud está constituido por una mezcla de derrubios donde predomina la fracción de los bloques y cantos (Gómez et al., 2003). No obstante, cabe destacar la presencia de abundante material fino que proviene de la alteración de los micaesquistos, que por su composición mineralógica resultan muy vulnerables a la meteorización (Puga et al., 2007). Esta fracción fina se distribuye en mayor proporción en los sectores occidental y central del talud. Los clastos acumulados en las repisas superiores acaban alterándose fácilmente gracias al agua de deshielo. Los finos resultantes son transportados por estas aguas hacia los sectores indicados del talud, a causa de la favorable inclinación NE-SW de las repisas y a la facilidad para infiltrarse entre los grandes bloques (Castillo y Fedeli, 2002).

Dentro del conjunto de los sistemas glaciares que se extendieron en Sierra Nevada durante el Cuaternario, el Corral del Veleta actuó como cabecera del glaciar del Guarnón, que con recorrido hacia el norte éste se prolongó en más de $6 \mathrm{~km}$ (Gómez et al., 2004 a,b). La favorable localización y disposición topoclimática del Corral permitió que durante la denominada Pequeña Edad del Hielo (siglos XV/mediados del XIX) también se formara un pequeño glaciar, aunque ahora recluido en su interior (Gómez et al., 2004a; Gómez y Plana, 2006). A mediados de la década del pasado siglo el glaciar terminó por desaparecer (Gómez et al., 1996). En la actualidad, por tanto, no existen masas de hielo glaciar en la superficie del Corral. Sin embargo, investigaciones recientes (prospecciones geoelétricas de 1995 y 1998) mostraron la existencia de hielo en el interior del suelo (GEOTECNIA DEL SUR, 1995; TERRADAT-LTD y ETH, 1998). Asimismo, la 
monitorización de la temperatura del suelo detectó igualmente la existencia de masas heladas internas (Gómez et al., 2001, 2003). Estos métodos han puesto de manifiesto la existencia de permafrost discontinuo en el tercio centro-oriental del talud. Por el contrario, en el sector occidental del Corral los resultados indican ausencia de permafrost. Esta situación se ha explicado por la retirada del hielo glaciar en sentido oeste-este en el interior del Corral, quedando recluido en el sector más centro-oriental en sus últimos años (García Sainz, 1947). A medida que el hielo glaciar se fue retirando, se formó el talud actual, aunque en sus primeras fases incluyendo bolsas de hielo en su interior, como todavía lo hace en su sector oriental (Gómez et al., 2003).

\section{Metodología}

En primer lugar se realizó la monitorización de la mínima permanencia anual de la nieve sobre el talud a partir del análisis y tratamiento informatizado de fotografías que se obtienen con cámara digital desde dos puntos fijos: el mirador del Corral, que permite captar una panorámica oblicua, y el borde extremo del Pico del Veleta, desde donde es posible obtener una fotografía vertical del fondo del circo. Las fotografías se realizan cada año a principios de septiembre, justo antes de que se inicien las primeras nevadas de la siguiente temporada. Una vez realizadas las fotografías éstas son tratadas informáticamente usando un programa de transformación de imágenes CAD MicroStation y su módulo Descartes, con lo que se consigue rectificarlas geométricamente, de modo que su información puede ser extraída y trasvasada al mapa topográfico a escala 1:10.000. Este tratamiento consiste en dos procesos: de una parte, las fotografías oblicuas de cada año se superponen utilizando como base la fotografía inicial (agosto de 1998). En esta fotografía se identifican una serie de puntos de control, los cuales se utilizarán para proceder a la rectificación de las fotografías de los otros años; de otra, las fotografías verticales tomadas desde el Pico se georreferencian con la ayuda de un CAD, utilizando para ello como base una ortofoto a escala 1:5000 (Ministerio de Economía y Hacienda, Dirección General del Catastro, Año 1998). Posteriormente se procede a la digitalización del recubrimiento de la nieve para cada verano, complementándose aquellas áreas no visibles con la información proporcionada por las fotografías oblicuas. De este modo, se ha obtenido el mapa del recubrimiento nival a finales del verano para cada uno de los últimos once años (1998-2008). Finalmente, el tratamiento en un Sistema de Información Geográfica de los diferentes mapas anuales de nieve ha permitido obtener un mapa final con las áreas de máxima permanencia estival de la nieve.

En segundo lugar, se ha realizado un análisis de los flujos superficiales que afectan al talud detrítico mediante la localización y cartografía geomorfológica precisa de los nuevos flujos aparecidos cada año, así como su caracterización morfométrica, morfológica y sedimentológica. A partir de esta monitorización se ha podido cuantificar el desplazamiento del material movilizado, así como su espesor y los rasgos granulométricos de las partículas removidas. Además, desde el año de su formación se ha realizado un seguimiento de su evolución, analizando los cambios sufridos en los años posteriores o constatando su desaparición debido a la superposición de nuevos procesos. 


\section{Resultados}

\subsection{La extensión y distribución de la cubierta de nieve a finales del verano en el Corral del Veleta}

La monitorización de la cubierta de nieve en el Corral del Veleta se inició de manera sistemática en el verano de 1998, precisamente el año en que su grado de recubrimiento en el suelo fue el mayor del período de estudio. (Fig. 3, 4 y 5). La evolución de la duración de la cubierta de nieve muestra una tendencia negativa, donde únicamente los años de 2001 y 2004 tuvieron una apreciable cubierta mínima de nieve, con un 65\% y un $27 \%$ de recubrimiento respectivamente con respecto a 1998, mientras que el resto de los años la cubierta mínima nival fue inferior del $10 \%$ con respecto a ese primer año. Esta tendencia negativa se agudiza especialmente desde 2005, no superando desde entonces la superficie mínima ocupada por la nieve el $2 \%$. Se puede comparar estos resultados con la cubierta de nieve mostrada por las fotografías aéreas de finales de julio de 1957, 1985 y 1990, y de septiembre de 1989 (Fig. 6 y 7), a pesar de no ser coincidentes las fechas en las primeras fotos. En todo caso, salvo la situación de 1990 donde el recubrimiento es similar a 1998, en el resto de años el Corral está completamente cubierto por la nieve.

\subsection{La existencia y localización de neveros semi-permanentes}

La integración de los once mapas individuales de la cubierta de nieve permite obtener un modelo aproximado del ritmo de la fusión nival y determinar las áreas donde la nieve tiende a concentrarse durante un tiempo más prolongado (Fig. 5). Este mapa muestra la persistencia de tres importantes neveros de larga duración en las áreas más protegidas de la base de la pared y el talud: el primero se emplaza en el sector más occidental del talud; el segundo se localiza en el sector central, enfrente de la Laguna del Corral, y presenta una forma estrecha y alargada, en torno a unos $120 \mathrm{~m}$ de longitud; el tercero se localiza algo más al oriente y se prolonga adosado a una repisa de la pared hasta enlazar con la ladera del cerro de Los Machos.

\subsection{La formación de movimientos en masa y su relación con los neveros semi- permanentes}

Durante el verano de 1998 no se observó ningún movimiento en el talud debido a la extensa cubierta mínima de nieve de ese año. En el verano de 1999 la cubierta de nieve tan sólo cubría un $9 \%$ respecto al año precedente. La mayor actividad del talud de ese año se observó frente al nevero instalado en su sector central (Fig. 8). En realidad, se trata del sector del talud detrítico de mayor inestabilidad geomorfológica pues en él concurren una serie de características favorables como son: su gran pendiente, la irregularidad del substrato rocoso, la gran abundancia de finos, el aporte hídrico constante proporcionado por la fusión nival y la existencia de niveles discontinuos de permafrost en un estado de conservación crítico. Todo esto favoreció que en el verano de 1999 se formaran varios deslizamientos rotacionales tras la fusión de un volumen excepcional de 


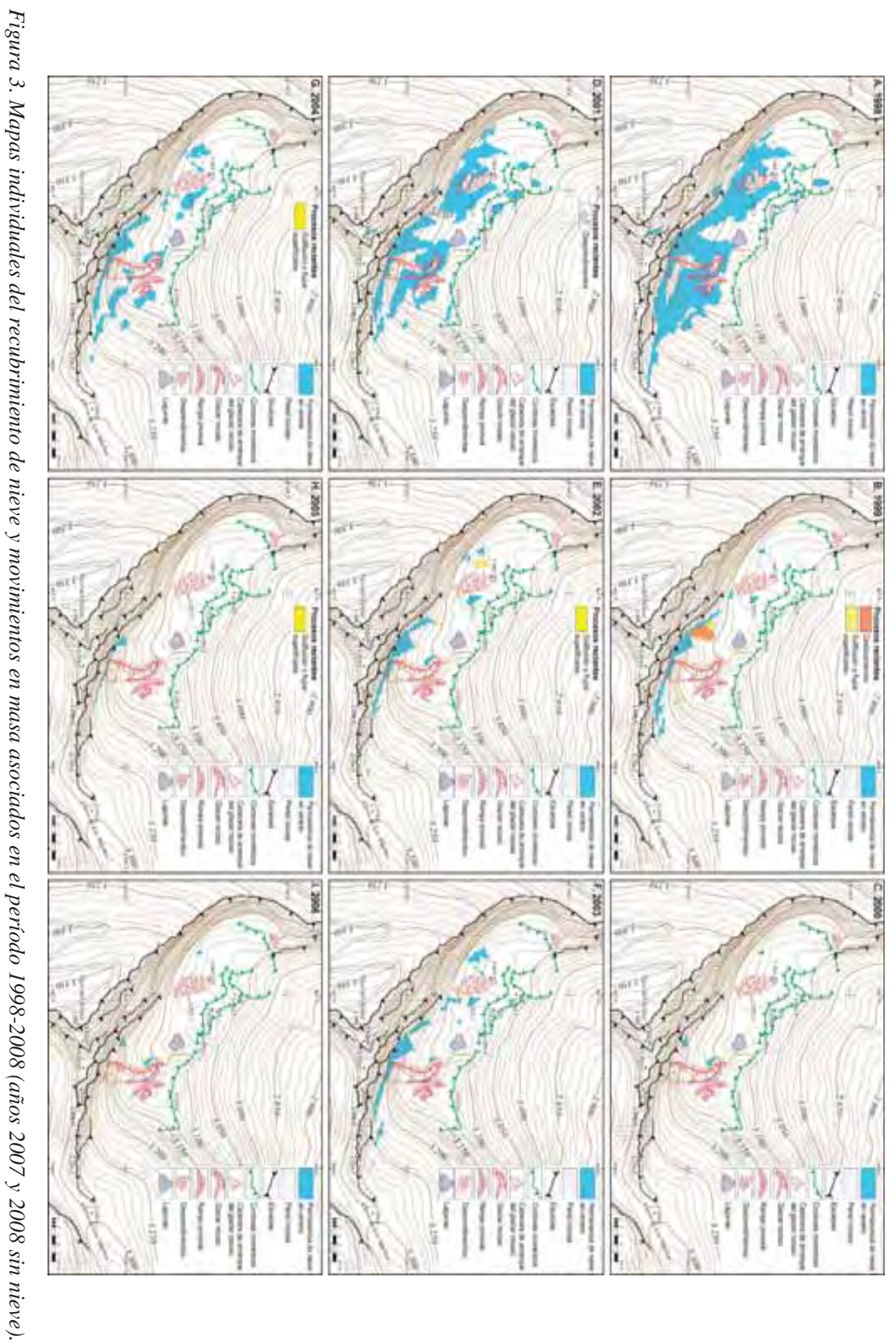




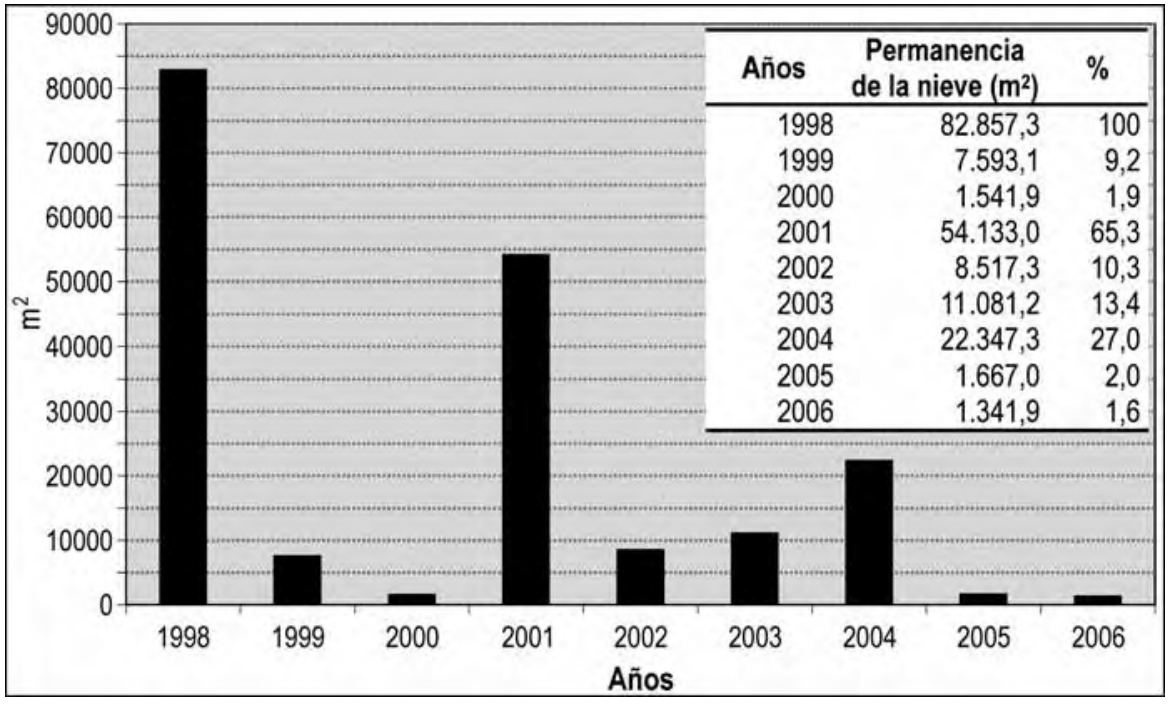

Figura 4. Evolución de la cobertura de nieve en el fondo del Corral del Veleta a finales del verano, superficie ocupada $\left(\mathrm{m}^{2}\right)$ y porcentajes $(\%)$ calculados tomando como referencia el verano de 1998 en el período 1998-2008 (años 2007 y 2008 sin nieve).

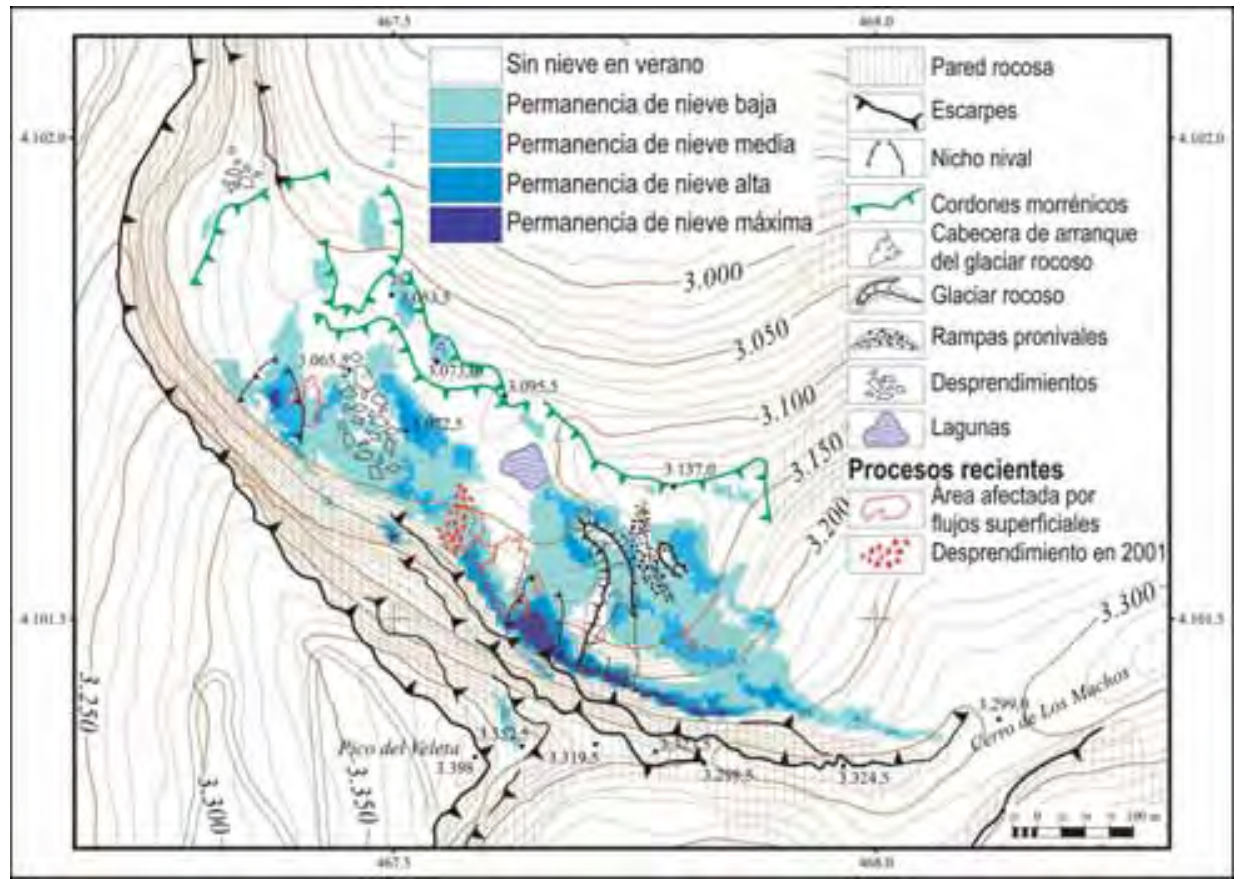

Figura 5. Distribución de la nieve en el Corral a partir de la integración de los mapas individuales en el período 1998-2008 (años 2007 y 2008 sin nieve). 


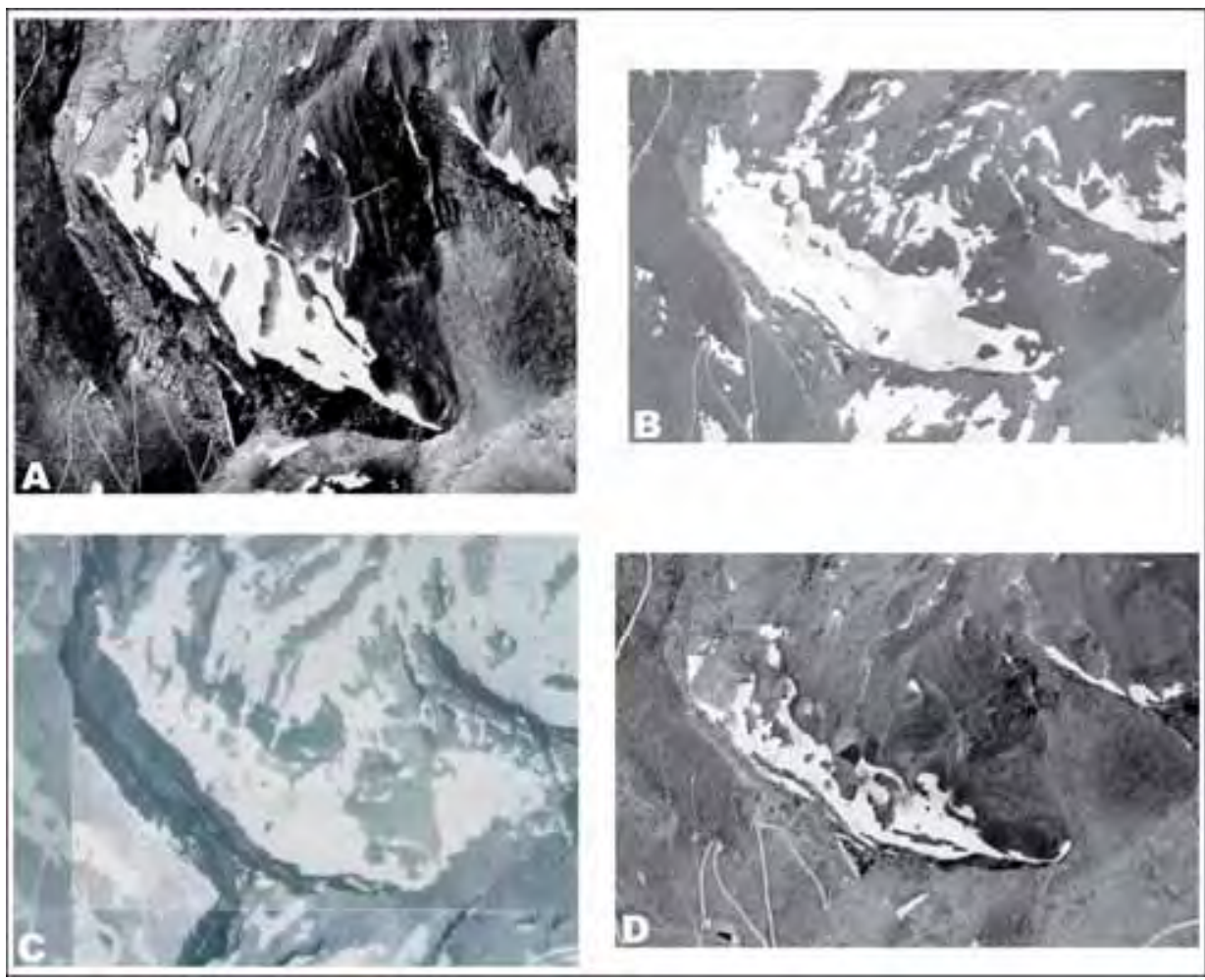

Figura 6. Fotografías aéreas que muestra la distribución de la nieve en el Corral del Veleta. A. Vuelo Americano, julio de 1957; B. IGN, julio de 1984; C. Ministerio de Hacienda, septiembre de 1989; D. Junta de Andalucía, julio de 1990.

nieve: aquella que perduró en 1998 más la caída durante el propio año de 1999, junto con la posible degradación de niveles internos de permafrost.

Algo similar ocurrió en el verano de 2002, fecha en la que se produjeron cuatro flujos superficiales (Fig. 8), pues la cubierta mínima nival en el año previo presentaba una apreciable extensión, mientras que al año siguiente se redujo a la pervivencia de los tres neveros descritos con anterioridad. Dos de los flujos se ubicaron junto al nevero del sector occidental del talud detrítico, donde el permafrost está ausente; mientras que los otros dos se localizaron frente a los neveros instalados en la parte central y oriental del talud, donde, -como se ha indicado anteriormente-, se ha constatado la existencia de niveles de permafrost.

Finalmente, varios flujos más se originaron en el año 2004 y 2005. Éstos se formaron de nuevo en el frente de los neveros del sector centro-oriental (Fig. 8). De nuevo, se generaron también tras el deshielo de abundante recubrimiento nival. Desde esta fecha no se han formado nuevos flujos superficiales. Sólo se han registrado movimientos anuales en el área afectada por los deslizamientos de 1999. 


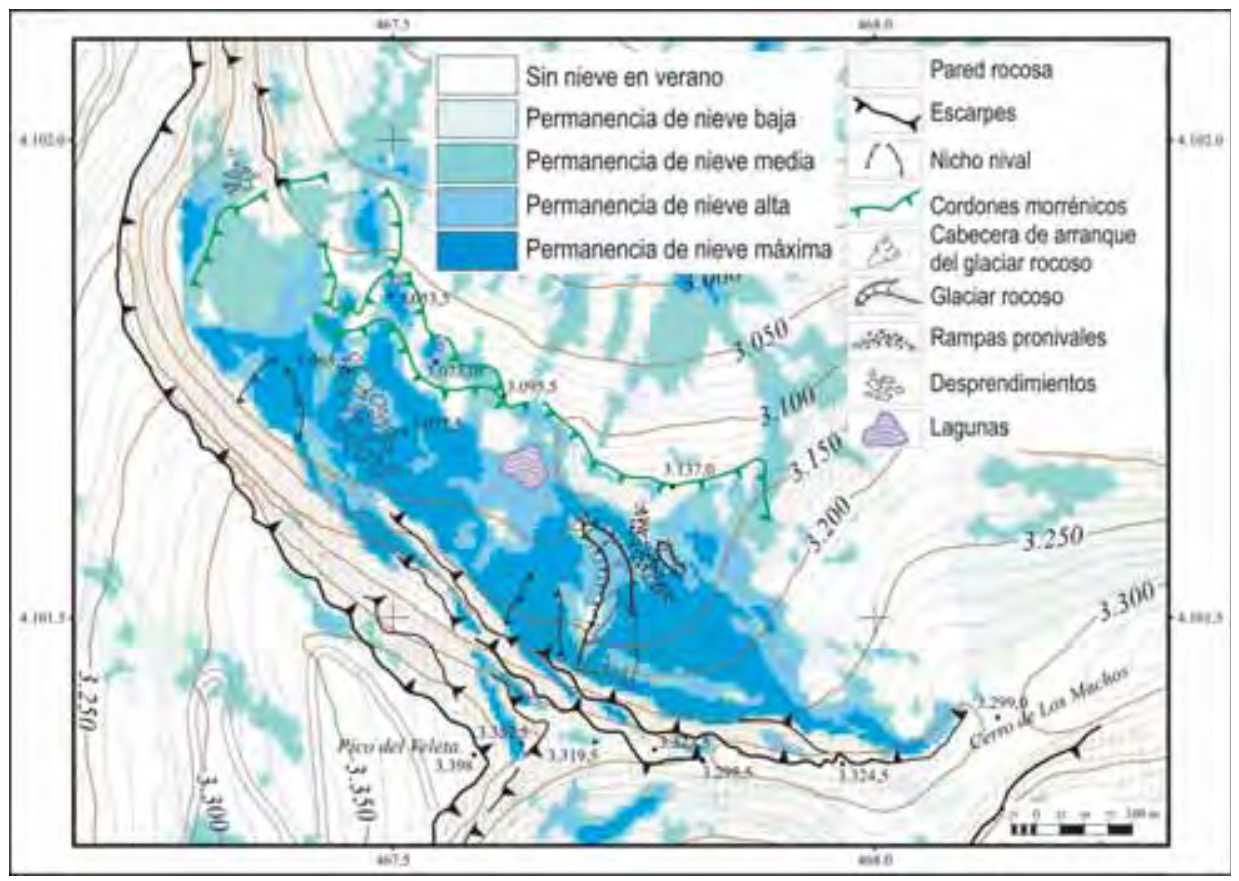

Figura 7. Distribución de la permanencia de la nieve a partir de la integración de las fotografías aéreas de la Figura 6.

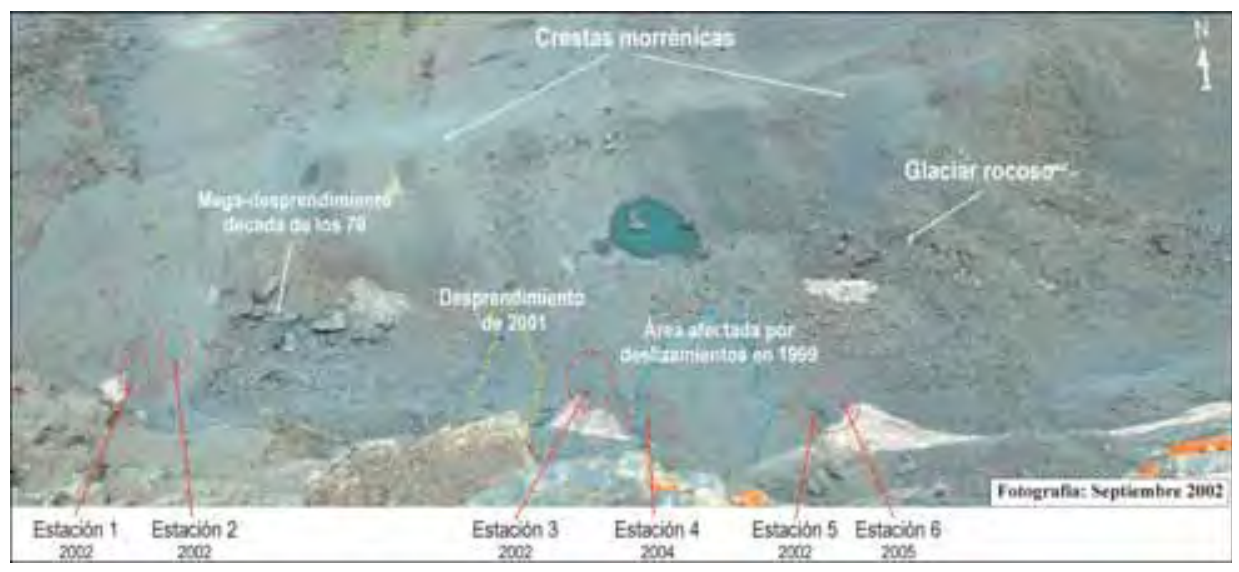

Figura 8. Fotografía vertical del conjunto del fondo del Corral tomada desde el pico del Veleta. Se muestra la localización de los flujos superficiales (Estación 1 a 6) y su año de formación. 


\subsection{La formación de flujos superficiales y sus características}

Como se ha indicado, frente al nevero situado en el sector occidental del talud detrítico se formaron en el año 2002 dos flujos superficiales (Fig. 8). Este sector del talud se caracteriza por sus reducidas dimensiones, por una pendiente regular inferior a los $20^{\circ}$ y por ausencia de permafrost en su interior. La sedimentología del talud está constituída mayoritariamente por cantos y gravas empastados en una abundante matriz fina, entre los que se intercalan bloques entre 20 y $40 \mathrm{~cm}$ de eje mayor.

De los dos flujos formados en 2002 el de mayores dimensiones (Estación 2, Fig. 9 y 10) tiene una longitud máxima de unos $50 \mathrm{~m}$ y una anchura variable entre 17 y 25 $\mathrm{m}$, lo que supone un área removida en torno a los $900 \mathrm{~m}^{2}$. El espesor del material movilizado es muy delgado, oscilando entre $30-40 \mathrm{~cm}$, y afecta básicamente a la fracción fina y a pequeños clastos de tamaño grava. La configuración morfológica del flujo muestra, en detalle, el desarrollo de numerosas y múltiples lenguas de pequeñas dimensiones interconectadas entre sí, que terminan formando diminutos lóbulos con frentes

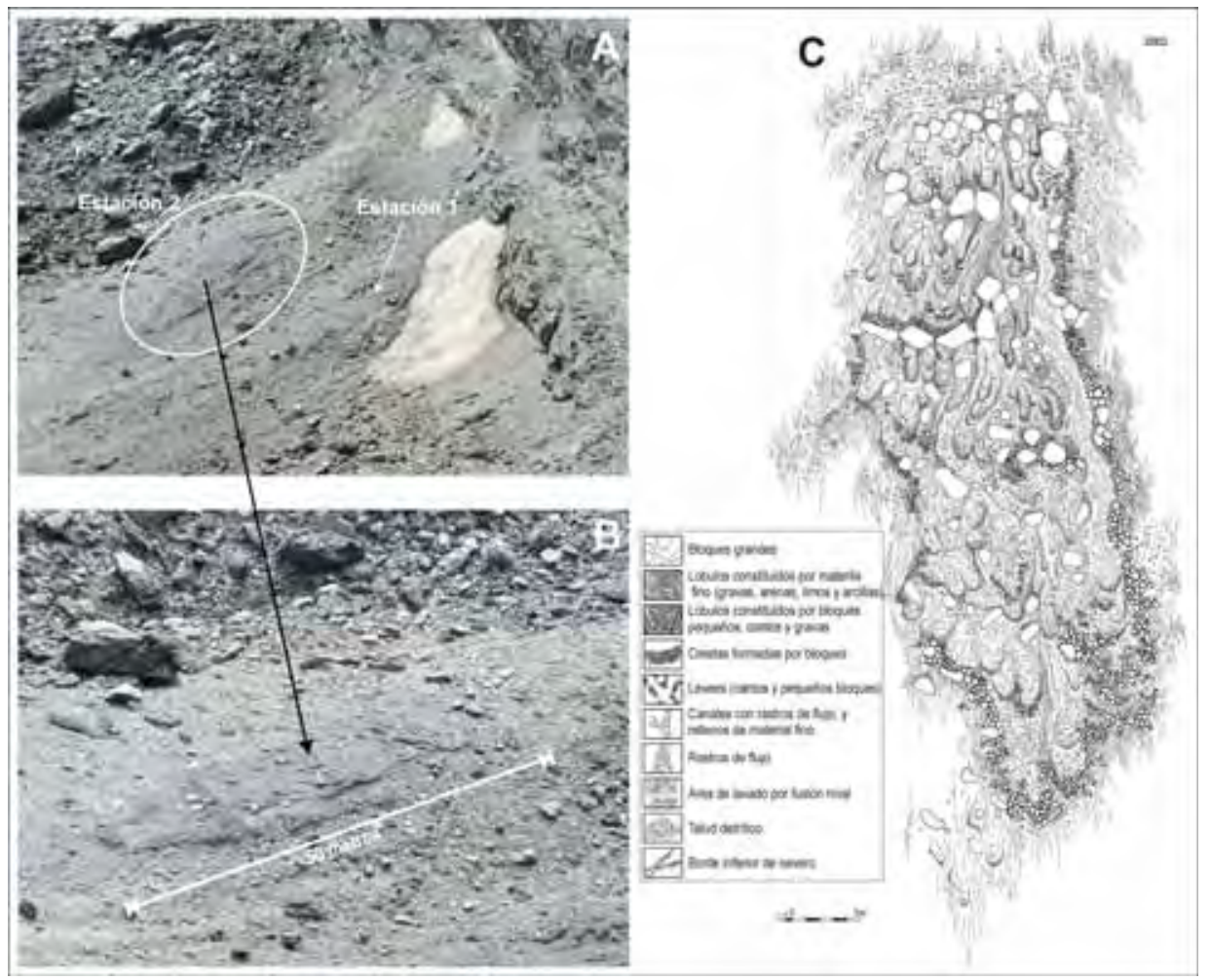

Figura 9. Fotografía oblicua de la localización de los flujos superficiales asociados con el nevero del sector occidental del talud detrítico $(A)$, detalle del flujo superficial correspondiente a la Estación 2 (ver figura 8) (B) y croquis geomorfológico (C). 

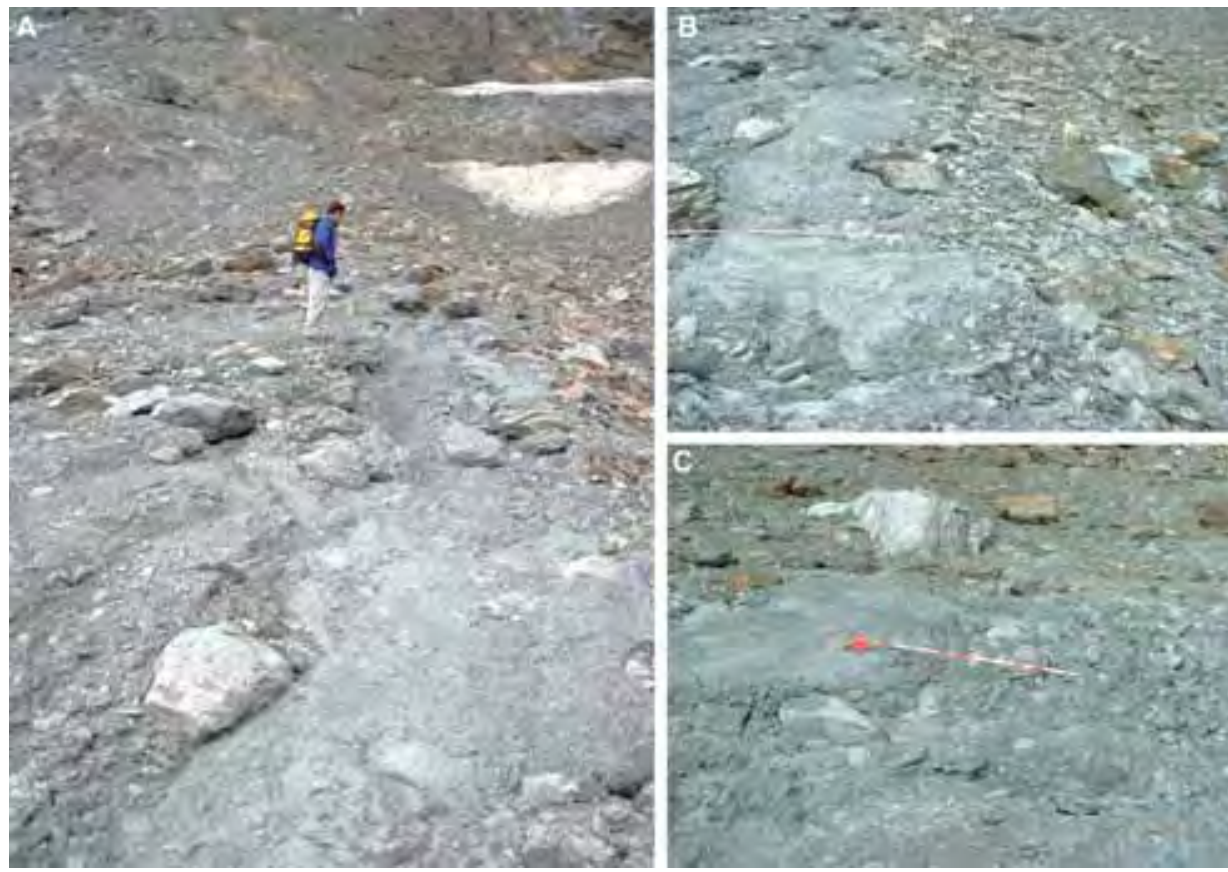

Figura 10. Vista general del flujo superficial correspondiente a la estación 2 (A) y fotografías de detalle de las lenguas lobuladas formadas por material fino $(B$ y $C)$.

arqueados. En la parte interna de los lóbulos se acumula el material fino (pequeñas gravas, arenas, limo y arcilla), a veces formando diminutos arcos. Este tipo de flujo es incapaz de desplazar los bloques de mayores dimensiones, por lo que su trayectoria tiende a desviarse o sus frentes quedan inmovilizados si el bloque es de gran tamaño. Sólo una lengua situada en el costado oeste del flujo, de unos $25 \mathrm{~m}$ de longitud, pudo descender en línea recta sin interrupciones. Desde su formación en 2002, este flujo superficial sigue conservando en gran medida su morfología y únicamente se han difuminado los lóbulos iniciales.

Otro flujo se formó también en el 2002, pero frente al nevero situado en el sector central del talud (Fig. 8). En este sector la pendiente media del ápice y del tramo intermedio suele oscilar entre los $28-30^{\circ}$, mientras que en la parte final aparece una ruptura de pendiente, aumentando considerablemente la inclinación $\left(40^{\circ}\right)$ al coincidir con el escalón formado por una repisa estructural. Aproximadamente el $60 \%$ de la masa de derrubios movilizada está constituida por bloques cuyo eje mayor supera los $25 \mathrm{~cm}$. El resto está formada por bloques menores, cantos y una abundante presencia de partículas de granulometría fina. Una campaña realizada en el invierno de 2000 detectó la presencia probable de permafrost en el interior de este talud central, especialmente en su tramo superior (Gómez et al., 2001, 2003, 2004 a,b). Este flujo tuvo un desarrollo longitudinal próximo a los 30 m y una anchura variable entre los 10 y 15 m (Estación 3, Fig. 11). El 
flujo se iniciaba con regueros ligeramente incididos por el agua de deshielo, hasta transformarse unos 10 m más abajo en una serie de lenguas estrechas en cuyo frente se formaban lóbulos de aspecto arqueado constituidos por cantos y bloques pequeños. La forma de este flujo desapareció debido al trabajo de procesos posteriores, pero en 2005 se formó otro flujo superficial situado un poco más al este del anterior, con una longitud de unos 14 m y una anchura próxima a los 6 m (Estación 4, Fig. 12). Se trata de varias lenguas coalescentes, cuyo desarrollo aparece condicionado por la presencia de grandes bloques (alguno superior a los 2,5 m de eje mayor), que desvían la dirección de los flujos o los detienen, para formar arcos lobulados.

Dos flujos superficiales también se formaron frente al nevero que se emplaza en el sector más oriental del talud detrítico. Se caracteriza este sector del talud por una pendiente media de $25^{\circ}$, por el predominio de bloques de gran tamaño, donde aproximadamente más del $50 \%$ tienen un eje mayor superior a $50 \mathrm{~cm}$, por la escasez de partículas finas en superficie, pues han emigrado al interior y hacia la base del talud por los procesos de lavado, y finalmente, por la presencia permafrost. Un primer flujo se formó en el año de 2001 (Estación 5, Fig.13). Se trata de una típica lengua de bloques que alcanza un desarrollo longitudinal de unos 18-20 m, de los cuales los últimos dos

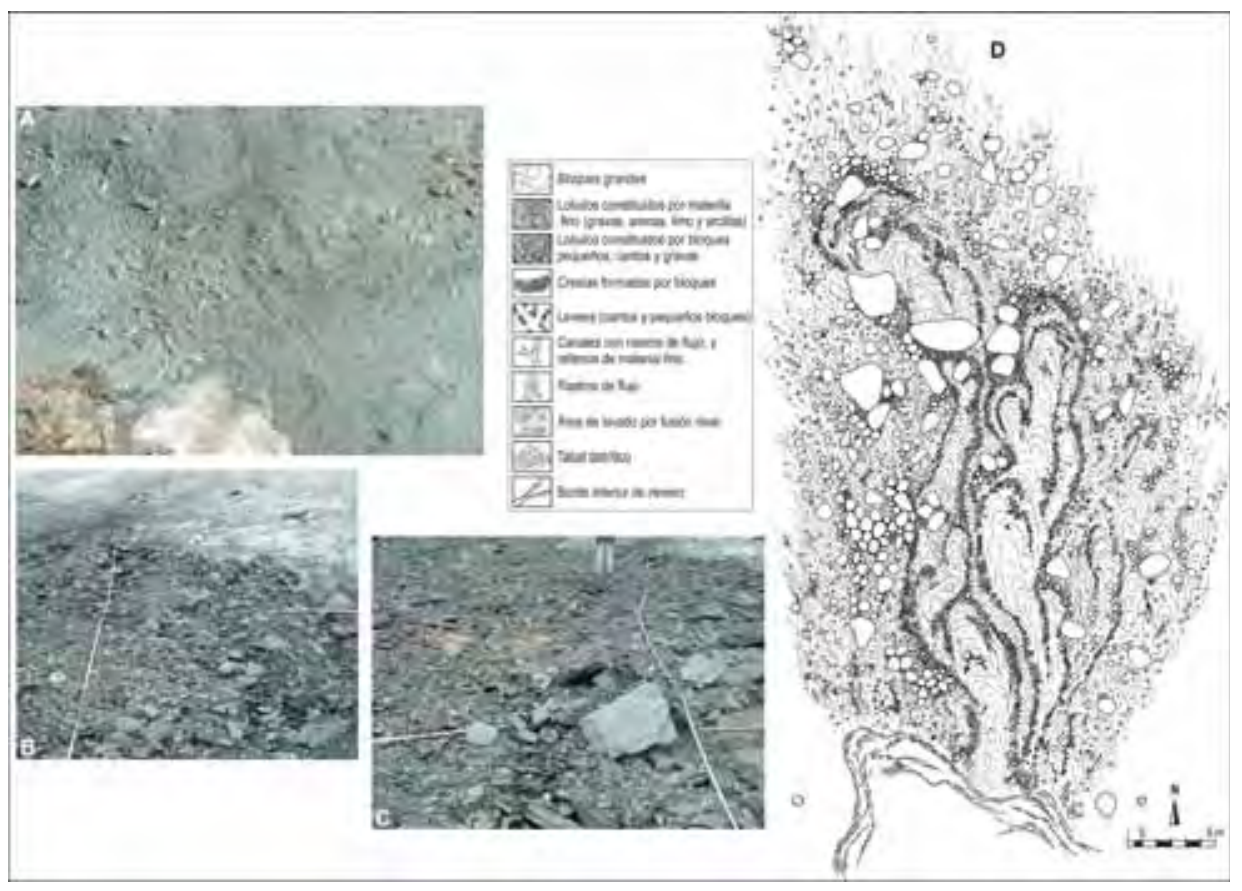

Figura 11. Flujo superficial asociado al nevero del sector central del talud detrítico correspondiente a la estación 3. Fotografía vertical del flujo (A), fotografías de detalle $(B$ y $C$ ) y croquis geomorfológico $(D)$. 


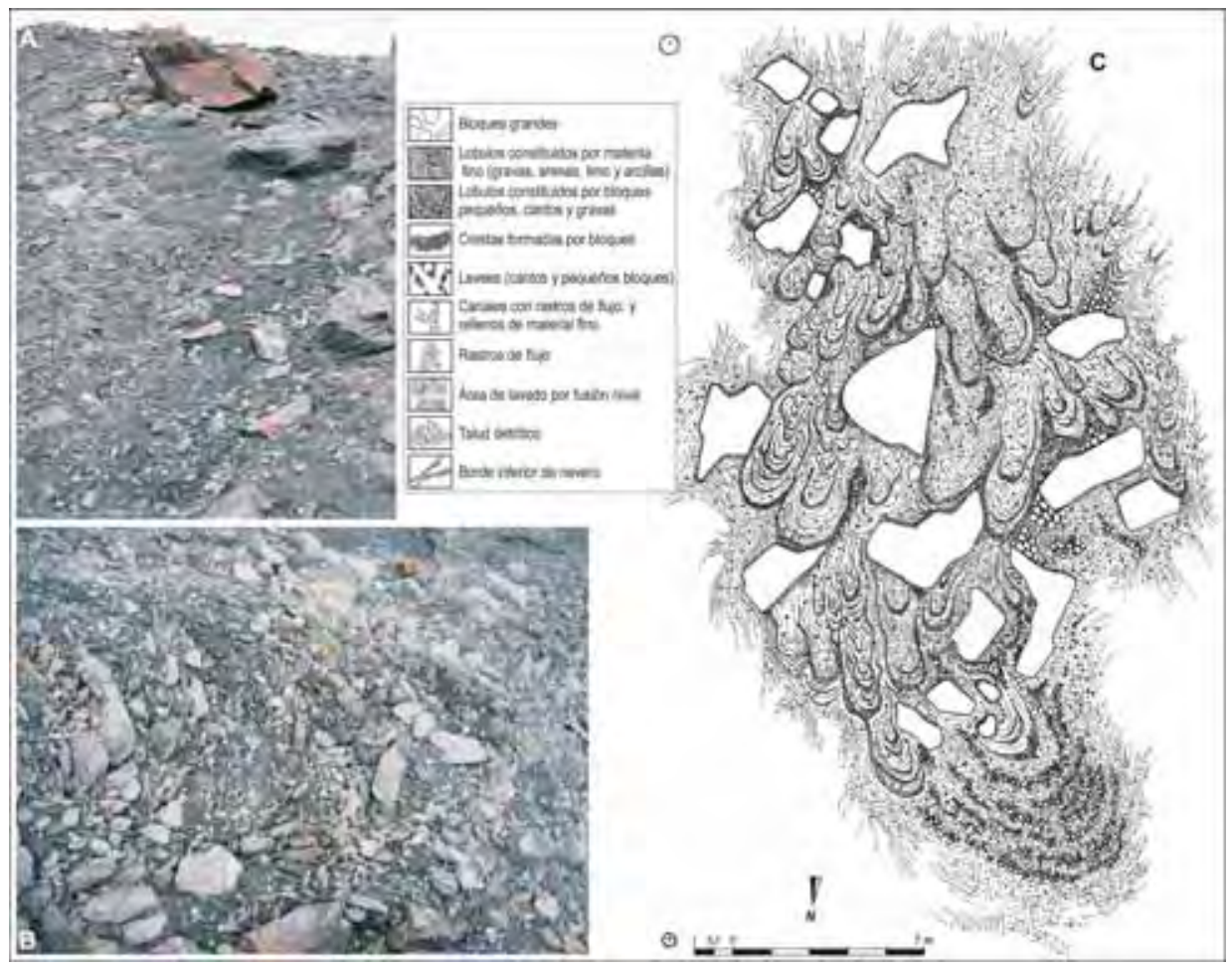

Figura 12. Flujo superficial asociado al nevero del sector central del talud detrítico correspondiente a la estación 4 (ver figura 8). Fotografías de detalle (A y B) y croquis geomorfológico $(C)$.

metros se corresponden con la formación de varios frentes arqueados constituidos por bloques menores en disposición vertical. El interior de la lengua, que tiene una amplitud entre 2 y 4 m, está rellenado por material fino, gravas y pequeños cantos. En los lados es frecuente encontrar pequeños lóbulos formados por material fino y pequeños cantos que quedan retenidos por bloques de mayores dimensiones. En 2005 se formó en este sector un nuevo flujo superficial. En este caso estaba integrado por lenguas menores que formaban crestas más o menos arqueadas de bloques pequeños y cantos. Por debajo de esta serie de lenguas se abría un estrecho canal adosado a la parte oriental del flujo, que canalizaba el drenaje subsuperficial de la fusión del nevero. Al igual que en los flujos del sector central, en estos orientales desaparecen sus formas de un año para otro. 


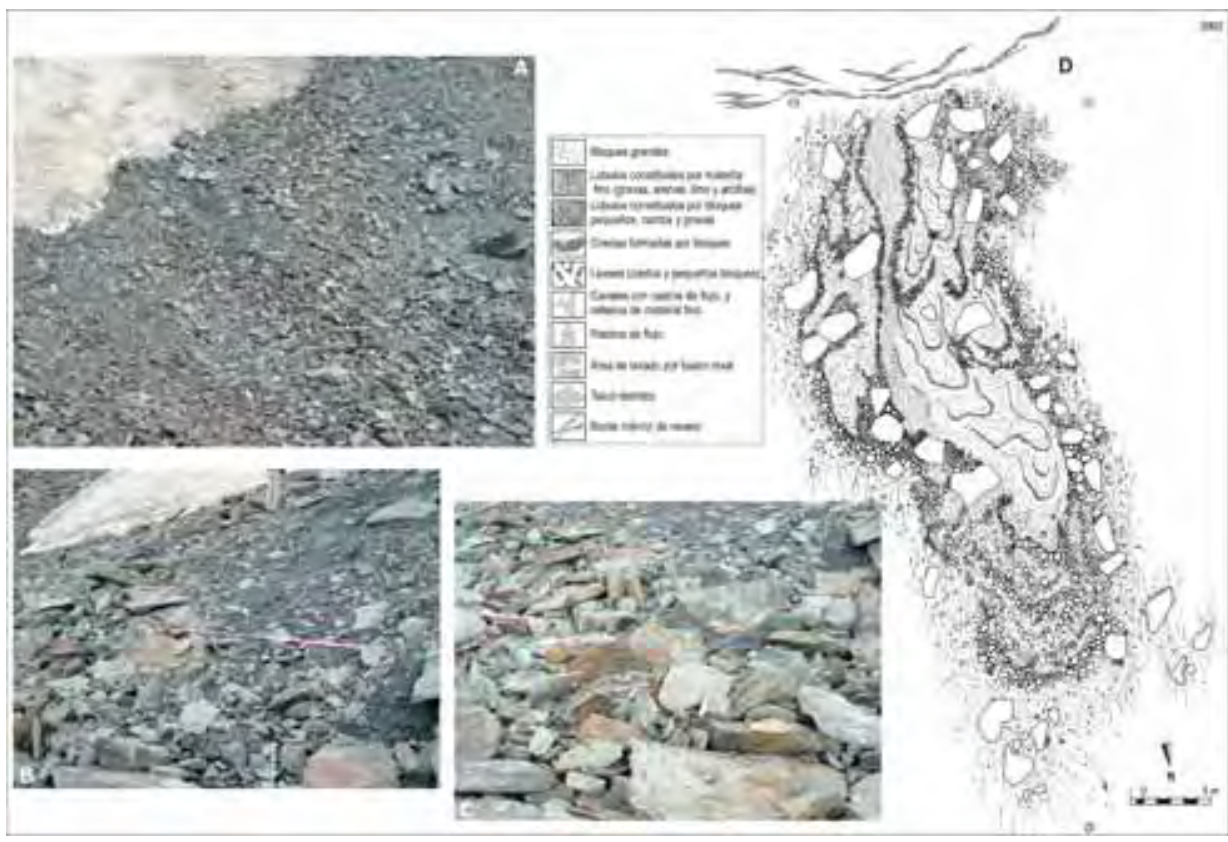

Figura 13. Flujo superficial asociado al nevero del sector oriental del talud detrítico correspondiente a la estación 5 (ver figura 8). Fotografía vertical del flujo (A), fotografías de detalle $(B$ y $C$ ) y croquis geomorfológico $(D)$.

\section{Discusión}

Los flujos superficiales (skinflows, según McRoberts y Morgenstern, 1974 y Lewkowicz, 1988) o movimientos en masa de similares características (sheet slides, según Rapp, 1960; solifluction sheets, según Christiansen, 1998; debris tongues, descritos por Jahn en 1967 y debris slides por Nyberg en 1985) es un tipo de proceso que se desencadena, según los autores citados, en las áreas pronivales con intensa ablación de los neveros durante el verano y donde el agua de deshielo empapa el suelo frente al nevero. Por lo tanto, el principal papel de la nieve consiste en suministrar el agua necesaria capaz de desestabilizar la masa de sedimentos. La presencia de material fino sin consolidar, relativamente impermeable, dificulta el drenaje en profundidad, favoreciendo, en gran modo, la formación de estos flujos. También favorece los flujos la disminución de la presión intergranular del suelo tras su deshielo, que al ser menor propicia que se sature sin dificultad en momentos de máximo aporte de agua por rápida fusión de la nieve (Matsuoka, 2002, 2009). La desestabilización afecta sólo a un nivel muy superficial pero a cambio le otorga una gran movilidad y capacidad de transporte, incluso con pendientes bajas. Estas condiciones están siendo óptimas en los últimos veranos en el talud de la pared septentrional del Pico del Veleta: deshielo del suelo, abundancia de agua de fusión nival, profusión de material fino relativamente impermeable por la presencia de arcillas 
provenientes de la alteración de los micaesquistos y práctica ausencia de vegetación. Además, los flujos superficiales estudiados se han formado básicamente durante los años en los que la fusión de la nieve ha sido prácticamente completa.

Junto al cometido fundamental de la nieve en la formación de los flujos descritos también ha debido jugar un papel muy importante la aceleración de la degradación y fusión del permafrost existente en el talud, pues además de suministrar agua puede constituir planos de despegue (McRoberts y Morgenstern, 1974; Harris, 1987; Lewkowicz, 1988; Christiansen, 1998). Esta degradación del permafrost se acelera cuando la cubierta de nieve no perdura durante los meses de verano. En este sentido, la mayor parte de los flujos superficiales estudiados se han desarrollado en el área pronival de neveros de fusión tardía localizados a su vez en los tramos superiores del talud detrítico del sector central y oriental del Corral del Veleta, donde se han detectado niveles de permafrost discontinuo en proceso de degradación (Gómez et al., 2001, 2003), muy probablemente acelerado en los últimos años por la menor persistencia de la nieve recubriendo el fondo del Corral durante los meses del estío. La mayor o menor importancia que desempeña la degradación del permafrost en la formación de los flujos superficiales en el talud del Veleta resulta en todo caso difícil de evaluar a falta de un estudio completo del régimen térmico del suelo. En el sector occidental del talud la formación de flujos resulta más restringida y diferente pues a la falta de permafrost en el interior del suelo hay que añadir el menor tiempo de duración de la nieve en superficie lo que limita el movimiento de la capa superficial del suelo y, por tanto, la mayor duración de los modelados creados.

\section{Conclusiones}

La evolución reciente de la duración y extensión de la cubierta nival en el Corral del Veleta a finales del estío en esta última década muestra una tendencia negativa que se manifiesta, a excepción de algunos años (1998, 2002, 2004), en que el grado de recubrimiento viene siendo menor, incluso inexistente. Sin embargo, tres neveros semi-permanentes permanecen casi todos los años recluidos en diferentes ámbitos del tramo superior del talud detrítico, instalado al pie de la pared norte del Pico del Veleta. En el frente de estos neveros se han producido diversos flujos superficiales de pequeñas dimensiones (longitudes entre los 20-50 m y anchuras entre los 15-20 m) aunque con capacidad para movilizar una delgada capa de derrubios de granulometría menor (cantos y partículas finas). Su morfología se caracteriza por la presencia de numerosas y cortas lenguas que terminan formando lóbulos. El interior de las lenguas está rellenado por las partículas finas y en los frentes se disponen de manera apilada e imbricada cantos y pequeños bloques.

Estos movimientos en masa han ocurrido, principalmente, cuando tras un año en el que la nieve recubría al final del verano gran parte del fondo del Corral, al siguiente año se producía la práctica fusión de ella, con la consiguiente liberación de un volumen de agua capaz de desestabilizar el suelo y originar el flujo. Pero también la formación de flujos se está desarrollando sin la presencia de nieve, como ocurre en estos últimos años. Ahora la causa principal es la degradación de niveles de permafrost en el interior del 
suelo motivada por la eficacia térmica de la propagación de la radiación exterior, tal como sucede en el sector centro-oriental del talud.

En definitiva, flujos superficiales inventariados en el seno de Corral del Veleta se desarrollan en sectores del talud donde el permafrost está ausente, por tanto su origen parece tener una clara relación con el deshielo nival. Además en estos casos, la morfología de los flujos una vez formados no se destruye. En cambio, en los sectores del talud donde se ha detectado la presencia de permafrost, las formas modeladas por los flujos superficiales desaparecen de un año para otro.

\section{Agradecimientos}

Esta investigación se ha realizado dentro del proyecto 018/2007 (Degradación de hielo fósil y permafrost y cambio climático en Sierra Nevada, España), financiado por la convocatoria de ayudas a proyectos de investigación en la Red de Parques Nacionales (Ministerio de Medio Ambiente, Rural y Marino).

\section{Referencias bibliográficas}

Akerman, H.J. (1984). Notes on talus morphology and processes in Spitsbergen. Geografiska Annaler, 66A (4): 267-284.

Andersland, O.B., LADANYI, B. (2004). Frozen ground engineering. John Wiley and Sons, Inc., Hoboken, New Jersey.

AndrÉs, N., PAlacios, D. (2004). Interrelación nieve/geomorfología en la sierra de Guadarrama: altas cuencas del Ventisquero de la Condesa y Valdemartín. Cuadernos de Investigación Geográfica, 30: 85-116.

Benedict, J.B. (1970). Downslope soil movement in a Colorado alpine region: rates, processes, and climatic significance. Arctic and Alpine Research, 2(3): 165-226.

CAINE, N. (1976). The influence of snow and increased snowfall on contemporary geomorphic processes in alpine areas. En: Ecological impacts of snowpack augmentation in the San Juan Mountains (Steinoff H.W., Ives J.D., Eds). Colorado State University, pp. 145-200, Fort Collins.

Castillo, A., Fedeli, B. (2002). Algunas pautas del comportamiento hidrogeológico de rocas duras afectadas por glaciarismo y periglaciarismo en Sierra Nevada (España). Geogaceta, 32: 195-197.

ChuecA, J., Julián, A. (2004). Influencia de la radiación solar en el proceso de degradación y fragmentación post-Pequeña Edad de Hielo del glaciar de la Maladeta (Pirineo central Español). Cuadernos de Investigación Geográfica, 30: 61-84.

Christiansen, H. H. (1998). Nivation forms and processes in unconsolidated sediments in Greenland. Earth Surf. Process. Landforms, 23: 751-760. 
FunK, M., Hoelzle, M. (1992). A model of potential direct solar radiation for investigating occurrences of mountain permafrost. Permafrost and Periglacial Processes, 3: $139-142$.

GARCía SAINZ, L. (1947). El clima de la España cuaternaria y los factores de su formación. Secretariado de Publicaciones. Universidad de Valencia. Valencia.

Geotécnica Del Sur (1995). Sondeos eléctricos verticales en el Corral del Veleta (Sierra Nevada, Granada). Report IG-1519, Granada.

Goodrich, E. L. (1982). The influence of snow cover on the ground thermal regime. Canadian Geotechnical Journal, 19: 421-432.

Gómez, A., Schulte, L., Salvador, F. (1996). Contribución al conocimiento de la deglaciación reciente y morfología asociada del Corral del Veleta (Sierra Nevada). Cadernos do Laboratorio Xeologico de Laxe, 21: 543-558.

Gómez Ortiz, A., Schulte, L., García Navarro, A., Palacios, D. (1999). Sobre la existencia de permafrost en Sierra Nevada. Significado geomorfológico y paleoclimático. En: Avances en el estudio del Cuaternario Español (Pallí Buxó, L, Roqué Pau, C., Eds). AEQUA-Universitat de Girona, pp: 181-186, Girona.

Gómez, A., Palacios, D., Ramos, M., Tanarro, L. M., Schulte, L, Salvador, F. (2001). Location of permafrost in marginal regions: Corral del Veleta, Sierra Nevada. Permafrost and Periglacial Processes, 12: 93-110.

Gómez, A., Palacios, D., Luengo, E., Tanarro, L.M., Schulte, L., Ramos, M. (2003). Talus instability in a recent deglaciation area and its relantionship to buried ice and snow cover evolution (Picacho del Veleta, Sierra Nevada, Spain). Geografiska Annaler, 85 A(2): 165-182.

Gómez Ortiz, A., Schulte, L., Salvador Franch, F., Palacios, D., Sanjosé Blasco, J. J., AtKinson, A. (2004a). Deglaciación reciente de Sierra Nevada. Repercusiones morfogénicas, nuevos datos y perspectivas de estudio futuro. Cuadernos de Investigación Geográfica, 30: 147-168.

Gómez, A., Palacios, D., Ramos, M., Casas, A., Sanjosé, J. J., Salvador, F., Schulte, L., Tanarro, L. M., Atkinson, A., Sánchez, S., Tapias, J. C., Zamorano, J. J., Tomé, P., Serrano, A. (2004b). Permafrost, evolución de formas asociadas y comportamiento térmico en el Corral del Veleta (Sierra Nevada, España). Últimos resultados. Boletín de la Real Sociedad Española de Historia Natural, 99 (1-4): 47-63.

Gómez Ortiz, A., Plana Castellví, J. A. (2006). La Pequeña Edad del Hielo en Sierra Nevada a partir de los escritos de época (siglos XIII-XIX) y su relación con el progreso de la geografía física y geomorfología española. Boletín de la Asociación de Geógrafos Españoles, 42: 71-98.

Gómez Ortiz, A., Salvador Franch, F., Sanjosé Blasco, J. J., Palacios Estremera, D., Schulte, L., AtKinson Gordo, A. (2008). Evolución morfodinámica de un enclave montañoso recién deglaciado: el caso del Corral del Veleta (Sierra Nevada), ¿consecuencia del Cambio Climático? Scripta Nova. Revista Electrónica de Geogra- 
fía y Ciencias Sociales. Vol. XII (270) (26). <http://www.ub.es/geocrit/sn/sn-270/sn270-26.htm>.

HAEBerli, W. (1973). Die Basis temperatur der winterlichen Schneedecke als möglicher Indikator für die Verbreitung von Permafrost in den Alpen. Zeitschrift für Gletscherkunde und Glazialgeologie, 9: 221-227.

HALl, K. (1985). Some observations on ground temperatures and transport processes at a nivation site in northern Norway. Norsk Geografisk Tidsskrift, 39: 27-37.

Harris, C., Vonder Mühll, D., Isaksen, K., Haeberli, W., Sollid, J. L., King, L., Holmlund, P., Dramis, F., Guglielmin, M., Palacios, D. (2003). Warming permafrost in European mountains. Global and Planetary Change 39, 215-225.

HARRIS, C. (1987). Mechanisms of mass movement in periglacial environments. En: Slope Stability (Anderson, M.G., Richards, K.S., Eds). John Wiley and Sons, pp. 531-559, Chichester.

Heggem, E. S. F., EtzelmüLler, B., Berthling, I. (2001). Topographic radiation balance models - sensitivity and application in periglacial geomorphology. Norsk Geografisk Tidsskrift - Norwegian Journal of Geography, 55: 203-211.

HoElzLE, M. (1996). Mapping and modelling of mountain permafrost distribution in the Alps. Norsk Geografisk Tidsskrift, 50: 11-15.

JAHN, A. (1967). Some features of mass movement on Spitsbergen slopes. Geografiska Annaler, 49A: 213-225.

Lewkowicz, A. G. (1988). Slope processes. En: Advances in Periglacial Geomorphology (Clark, M. J., Ed). John Wiley and Sons, pp. 325-368, Chichester.

MatsuoKa, N. (2002). Solifluction rates, processes and landforms: a global review. Earth Science Reviews, 55: 107-134.

Matsuoka, N. (2009). Dynamics of solifluction lobes in the Swiss Alps: A summary of 14-year monitoring. Geophysical Research Abstracts, 11, EGU2009-6473.

Mcroberts, E. C., Morgenstern, N. R. (1974). The stability of the thawing slopes. Canadian Geotechnical Journal, 11: 447-469.

NyBERG, R. (1991). Geomorphic processes at snowpatch sites in the Abisko mountains, northern Sweden. Zeitschrift für Geomorphologie N.F., 35(3): 321-343.

Palacios, D., De Andrés, N., Luengo, E. (2003). Distribution and effectiveness of nivation in Mediterranean mountains: Peñalara (Spain). Geomorphology, 54: 157-178

Puga, E., DíAz, A., Fontboté, J. M. (1974). Sobre la individualización y sistematización de las unidades profundas de la Zona Bética. Estudios Geológicos, 30: 543-548.

Puga, E., Díaz De Federico, A., Nieto, J. M., Díaz Puga, M. A. (2007). Petrología. Evolución geodinámica y georrecursos del Espacio Natural de Sierra Nevada. Estudios Geológicos, 63 (2): 19-40. 
RAPP, A., NYBERG, R. (1988). Mass movements, nivation processes and climatic fluctuations in northern Scandinavian mountains. Norsk Geografisk Tidsskrift, 42: 245-253.

RAPP, A. (1960). Recent development of mountain slopes in Kärkevagge and surroundings, Northen Scandinavia. Geografiska Annaler, 42A(2-3): 1-199.

SANZ de GALdEANO, C., LóPEZ, A. C. (1999). Nature and impact of the neotectonic deformation in the western Sierra Nevada (Spain). Geomorphology, 30(3): 259-273.

Soderman, G. (1980). Slope processes in cold environments of northern Finland. Fennia, 158: $83-152$.

STRÖMQUIST, L. (1985). Geomorphic impact of snowmelt on slope erosion and sediment production. Zeitschrift für Geomorphologie N. F., 29: 129-138.

Terradat Ltd \& ETH (1998). Terradat and ETH. (1998). Geophysical Survey Report. Permafrost Investigation. Veleta and Mulhacen, Sierra Nevada. Permafrost and Climate in Europe (PACE). Cardiff, Zürich.

White, S. E. (1981). Alpine mass movement forms (non-catastrophic). Arctic and Alpine Research, 13: 127-37. 